\title{
Les concepts en mouvement : une méthode basée sur la poésie pour la visite de groupe dans l'exposition d'art contemporain
}

\author{
Marie-Hélène Lemaire, Fondation PHI pour l'art contemporain \\ mhlemaire@phi.ca
}

Résumé : L'objectif de cet article consiste à présenter ma méthode de recherche des concepts en mouvement basée sur la poésie pour la visite de groupe dans l'exposition d'art contemporain. Cette méthode s'élabore en dialogue avec l'approche pédagogique de l'équipe de l'éducation à la Fondation PHI qui s'ancre dans la notion de mouvement et dans la méthodologie des concepts migratoires (Bal, 2002). Ma pédagogie féministe basée sur les concepts en mouvement est une extension des concepts migratoires, mais elle s'en distingue en se situant spécifiquement dans l'écriture poétique née d'une fulgurance de conscience-lucidité qui connecte mon corps-sujet avec l'affect de l'œuvre.

Mots-clés : Recherche poétique; Art contemporain; Éducation à l'art; Pédagogie du mouvement; Philosophie féministe; Corporéité; Poésie comme pédagogie.

Introduction

T 'objectif de cet article consiste à élaborer ma méthode de recherche des concepts en mouvement basée sur la poésie comme étape essentielle à ma préparation pour la visite de groupe dans l'exposition d'art contemporain. En tant que responsable de l'éducation à la Fondation PHI pour l'art contemporain, la poésie constitue pour moi la modalité de réflexion qui me permet de me connecter avec une empathie profonde avec les œuvres et les thématiques proposées par l'exposition, démarche nécessaire à mon enseignement subséquent lors des visites de groupe. Depuis 2011, je développe les concepts en mouvement dans le cadre du département de l'éducation de la Fondation PHI. Cette méthode s'élabore en dialogue avec l'approche pédagogique partagée de l'équipe de l'éducation, qui se base sur la notion de mouvement: intellectuel, affectif, émotif et physique. Au cœur de cette approche se trouve la méthodologie des concepts migratoires, qui s'inspire de celle des travelling concepts de la théoricienne culturelle et littéraire Mieke Bal (2001). ${ }^{1}$

Pour cet essai, j'offrirai un exemple de la manière dont ma méthode de recherche féministe des concepts en mouvement se déploie en offrant comme étude de cas l'exposition de l'artiste slovène Jasmina Cibic Everything That You Desire and Nothing That You Fear, qui a eu lieu à la

\footnotetext{
${ }^{1}$ Mes recherches sont soutenues et financées par la Fondation PHI pour l'art contemporain. Je tiens à remercier profondément la directrice générale et commissaire Cheryl Sim, ainsi que la directrice et fondatrice Phoebe Greenberg. Cet article constitue une branche d'un projet de recherche intitulé Expérimenter l'interprétation : gestes, mouvements, dialogue que j'ai co-mené avec ma collègue Pohanna Pyne Feinberg dans l'exposition de Jasmina Cibic Everything That You Desire and Nothing That You Fear en 2018 et 2019. Pohanna Pyne Feinberg est ma constante et indispensable interlocutrice pour mes recherches et pour cela je la remercie chaleureusement. Mes réflexions ont également été enrichies par les dialogues qui ont eu cours parmi l'équipe de l'éducation de la Fondation PHI : Amanda Beattie, Daniel Fiset et Tanha Gomes. Un grand merci !
} 
Fondation PHI du 25 octobre 2018 au 3 mars 2019. D'abord, pour décrire le contexte dans lequel je travaille, je vais présenter la pédagogie partagée de l'équipe de l'éducation à la Fondation PHI. Ensuite, je vais offrir une description de la méthode de recherche poétique que j'emploie. Par la suite, je présenterai les dimensions théoriques et conceptuelles générales de l'exposition de Jasmina Cibic, avec un accent placé sur l'une des installations de l'exposition intitulée The Land of Plenty (2018-2019). Puis, je présenterai mon poème Joséphine dans le boudoir de l'architecte, mon interprétation empathique de The Land of Plenty (2018-2019). Je ferai ensuite une analyse de mon poème qui me mènera en dernière partie à proposer pour le futur une intégration performative de la poésie lors de mes visites de groupes guidées.

\section{Une pédagogie du mouvement : concepts migratoires et concepts en mouvement}

Chaque année, le département de l'éducation de la Fondation PHI se met au service de milliers de participant $\cdot e \cdot s$ issu $\cdot e \cdot s$ du monde scolaire, académique, communautaire, des écoles de langues et, aussi, du grand public. Nous offrons des ateliers de création, des événements, des projets spéciaux, des collaborations et des visites de groupe. L'approche pédagogique partagée de l'équipe de l'éducation pour les visites de groupe dans l'exposition d'art contemporain est ancrée dans la pédagogie du mouvement et dans une réflexivité analytique qui s'appuient sur des points de vue divers. La question de l'accessibilité à l'art contemporain est essentielle pour la Fondation PHI et les éducateur-rice's se mettent au service de celle-ci en se basant sur la notion de mouvement : intellectuel, affectif, émotif et physique (Beattie et al., 2018). Au cœur de notre pédagogie du mouvement, se trouvent nos concepts migratoires qui sont une adaptation des travelling concepts de Mieke Bal et qui s'inspirent de la philosophie comme activité de création de concepts de Gilles Deleuze et de Félix Guattari. Selon ceux-ci, « les concepts sont des centres de vibrations, chacun en lui-même et les uns par rapport aux autres. C'est pourquoi tout résonne, au lieu de se suivre ou de se correspondre » (2005 [1991], p. 28). Selon Bal, les travelling concepts proposent un engagement envers l'art par l'entremise d'un corps résonant et vibratile, mais ils proposent également une mise à distance réflexive et analytique. Elle affirme : " the closer the engagement with the work of art, the more adequate the result of the analysis will be, both in terms of that particular artwork and as an account of the process of looking» (2001, p. xiii). Elle ajoute : «concepts can become a third partner in the otherwise totally unverifiable and symbiotic interaction between critic and object» $(2002$, p. 23).

Ma pédagogie féministe basée sur les concepts en mouvement est une extension des concepts migratoires, mais elle s'en distingue en se situant nécessairement dans l'écriture poétique. Alors que les concepts migratoires sont constitués de concepts résonants, vibratiles et analytiques, sélectionnés et utilisés par l'équipe de l'éducation, mes concepts en mouvement, tirés en partie de ces concepts migratoires, sont des mots qui sont issus d'une fulgurance de conscience-lucidité qui connecte mon corps-sujet avec l'affect de l'œuvre. Ainsi, alors que les mots qui composent mon poème possèdent des qualités de résonance tout comme les concepts migratoires, ils s'en distinguent par leur intensité, car ils possèdent aussi des qualités de fulgurance et de jaillissement. Également, mes concepts en mouvement se doivent de s'amalgamer, se façonner, s'agglomérer dans l'écriture poétique pour finalement se cristalliser dans le poème.

Au sein de l'équipe du département de l'éducation de la Fondation PHI, nous élaborons notre méthodologie des concepts migratoires depuis 2011. J'ai moi-même introduit cette approche interdisciplinaire dans l'équipe, que j'ai découverte et approfondie lors de mes études doctorales en communication à l'Université Concordia. À chaque fois que nous concevons notre programme éducatif pour la visite de groupe pour l'exposition à venir, nous tenons d'abord une séance de 
remue-méninges où tous les membres de l'équipe contribuent à la sélection et à la création d'une constellation de concepts migratoires partagés issus de nos lectures, de nos recherches et de notre expérience. Cet échange de remue-méninges parmi l'équipe avant l'ouverture de l'exposition est ouvert et improvisé et l'objectif est de créer à nouveau cet esprit et cette atmosphère lors de nos animations de visites de groupe. Lors de cette séance, les concepts migratoires sont colligés soit au crayon feutre sur un grand tableau blanc dans notre bureau ou bien dans un document Google partagé. Ceux-ci nous accompagneront durant toute l'exposition. D'autres séances de travail et de remue-méninges suivront bien sûr tout au long de l'exposition. Il est à noter donc qu'au département de l'éducation de la Fondation PHI, les éducateur·rice·s ne suivent pas un scénario de visite précis pour leur enseignement lors de la visite de groupe. Les concepts migratoires représentent une organisation sous forme de constellation informelle et mobile que les éducateur'rice's mettent en jeu en préparation de visites, durant l'animation des visites et en postvisites, et ce sans cesse, dans une logique cyclique d'impermanence et de rétroaction.

L'interprétation en mouvement d'une exposition d'art contemporain lors d'une visite de groupe est pour toutes et tous une sorte de voyage dans des espaces ouverts, imprévus, improvisés, fluctuants, expérimentaux, peut-être même obscurs et déstabilisants. Dans ce type d'espace, les concepts migratoires nous guident en formant des constellations : c'est en fonction des idées et des questions apportées par les participant $\cdot e \cdot s$ lors de la visite que certains concepts se dégageront et seront élaborés plus avant, alors que d'autres resteront temporairement dans l'ombre. À chaque nouvelle visite de groupe, selon le public qui y participe et l'éducateur rice qui anime, certains concepts migratoires changent ou reviennent et sont mis en jeu de manière différente. L'objectif lors de la visite de groupe consiste à créer des espaces ouverts, de soin et d'écoute, où les visiteur·euse's seront le plus possible libres de s'exprimer, de se mouvoir, de ressentir, de se mettre mutuellement au défi, et de développer leur esprit réflexif et analytique.

À partir de ce tronc commun de notre pédagogie du mouvement, chaque membre de l'équipe développe son programme éducatif selon ses spécialités de recherche, ses intérêts et son expérience de vie personnelle et professionnelle. Pour ma part, ma formation académique et mon expérience vécue m'ont menée vers un intérêt plus poussé pour le mouvement de l'affect et du corps. Ma pédagogie interdisciplinaire prend sa source dans ma formation universitaire en littératures française et québécoise (BA, Université Laval ; Université Paul-Valéry - Montpellier 3), en muséologie (MA, UQAM) et en communication (Ph.D., Université Concordia). Mais aussi, j'élabore cette approche depuis plus de vingt ans, alors que j'ai travaillé comme éducatrice dans diverses institutions muséales d'art contemporain à Montréal (Musée d'art contemporain de Montréal, Galerie Leonard et Bina Ellen de l'Université Concordia) et alors que je travaille, depuis 2011, au département de l'éducation de la Fondation PHI, et depuis 2020 en tant que responsable du département. Ainsi, toutes ces années à animer des visites de groupes ont développé ma conscience, sensibilité et connaissance des matérialités et résonances des œuvres et de l'exposition d'art contemporain, en déambulation avec d'autres corps. C'est pour tenter de comprendre ce phénomène d'interprétation collective incorporée, improvisée et dialogique de l'exposition d'art contemporain que j'ai fait un doctorat en communication. L'écriture poétique des concepts en mouvement est pour moi une extension de cette approche : un rapport somatique empathique et kinesthésique avec les matérialités, vibrations et énergies des œuvres, de l'exposition, des participant $\cdot e \cdot s$ à la visite, ainsi que des mots et des images.

Alors que les concepts migratoires représentent une constellation de concepts résonants et analytiques, les concepts en mouvement représentent un amalgame poétique de mots qui se doit d'aller au bout du geste jusqu'à la cristallisation. Mon processus consiste donc, après la séance de 
remue-méninges initiale de l'équipe, à placer mon corps en état de disponibilité et d'écoute à une force de fulgurance liée à la matière de quelques mots et d'images. Le poème commence généralement avec quelques mots qui émergent avec intensité et qui composent quelquefois un titre. Par la suite, j'amorce l'écriture en couchant assez rapidement, sous forme de collage, des grands jets de mots et d'images sur la page, qui s'organisent déjà en strophes. À cette première étape donc, les mots s'organisent en agglomérats poétiques. Et c'est ici que commence mon travail d'empathie envers l'œuvre que j'interprète par l'entremise des mots dans leurs qualités matérielles : textures, résonances, tonalités, rythmes, pressions, musicalité. Phénoménologue du geste, Vilém Flusser (2014) conçoit l'écriture comme un geste du corps. À son avis, celui ou celle qui écrit presse une virtualité cachée en lui ou elle vers l'extérieur, en traversant de nombreuses couches de résistance. Selon Flusser, pour parler d'écriture, il faut décrire la résistance des mots. Les mots sont des unités qui vibrent et possèdent une vie propre. Ils ont des rythmes, des harmonies, des mélodies, et avant toutes choses l'écrivain·e doit les écouter (2014, p. 22). J'ajoute à ces propos de Flusser que mon expérience de l'écriture poétique consiste à façonner et à modeler une matière informelle et amorphe avec mes mains, où mon esprit se laisse guider en se connectant à celles-ci, jusqu'à ce que la matière se cristallise.

\section{Présentation de l'exposition Everything That You Desire and Nothing That You Fear}

L'exploration de ma méthode poétique des concepts en mouvement pour la visite de groupe a eu lieu dans l'exposition de Jasmina Cibic : Everything That You Desire and Nothing That You Fear présentée à la Fondation PHI du 25 octobre 2018 au 3 mars 2019. Cibic est une artiste slovène qui réside à Londres. Au cœur de son travail se trouve le concept de 'soft power', force de persuasion, telle qu'exercée par les États-Nations. La force de persuasion est un type de pouvoir qui s'exerce par des moyens de séduction et d'attraction, plutôt que de coercition. L'exposition de Cibic était conçue comme une grande installation immersive composée de films, photographies, murales, arts textiles, sculptures, maquettes architecturales et performances. L'exposition portait sur la construction de la culture nationale à des fins politiques dans le cadre des expositions universelles au 20e siècle, en prenant pour étude de cas l'ex-Yougoslavie. Comment les gouvernements instrumentalisent-ils la culture pour façonner l'identité et la représentation nationale ? Comment l'architecture moderniste a-t-elle été utilisée par l'État de l'ex-Yougoslavie afin de participer au projet d'édification de la Nation et afin de projeter une image de domination suprême sur la scène internationale ? Et aussi : comment le corps de la femme est-il traité dans ces architectures patriarcales? Dans son exposition, Cibic s'intéressait, entre autres, à trois pavillons nationaux présentés par la Yougoslavie lors de trois expositions universelles : celui de Barcelone en 1929, celui de Bruxelles en 1958 et celui de Montréal en 1967. Expo 67 est la dernière exposition universelle où l'ex-Yougoslavie a présenté un pavillon, préfigurant sa dissolution dans les années 1990.

Dans la première salle d'exposition, où se trouvait l'installation The Land of Plenty (20182019), on pouvait voir The Pavilion (2018), une interprétation en petit format par Cibic du pavillon moderniste de l'Exposition universelle de Barcelone de 1929, conçu par l'architecte serbe Dragiša Brašovan. Ce pavillon représentait l'incarnation d'un nouveau pays, le Royaume des Serbes, des Croates et des Slovènes, créé en 1918. Cette maquette-pavillon se situait au centre d'une pièce entourée de rideaux, conçue par Cibic comme le boudoir d'un collectionneur anonyme ayant rassemblé divers artefacts tirés de la présence yougoslave à l'Exposition universelle de Barcelone. 


\section{Figure 1.}

The Land of Plenty (2018-2019) de Jasmina Cibic.

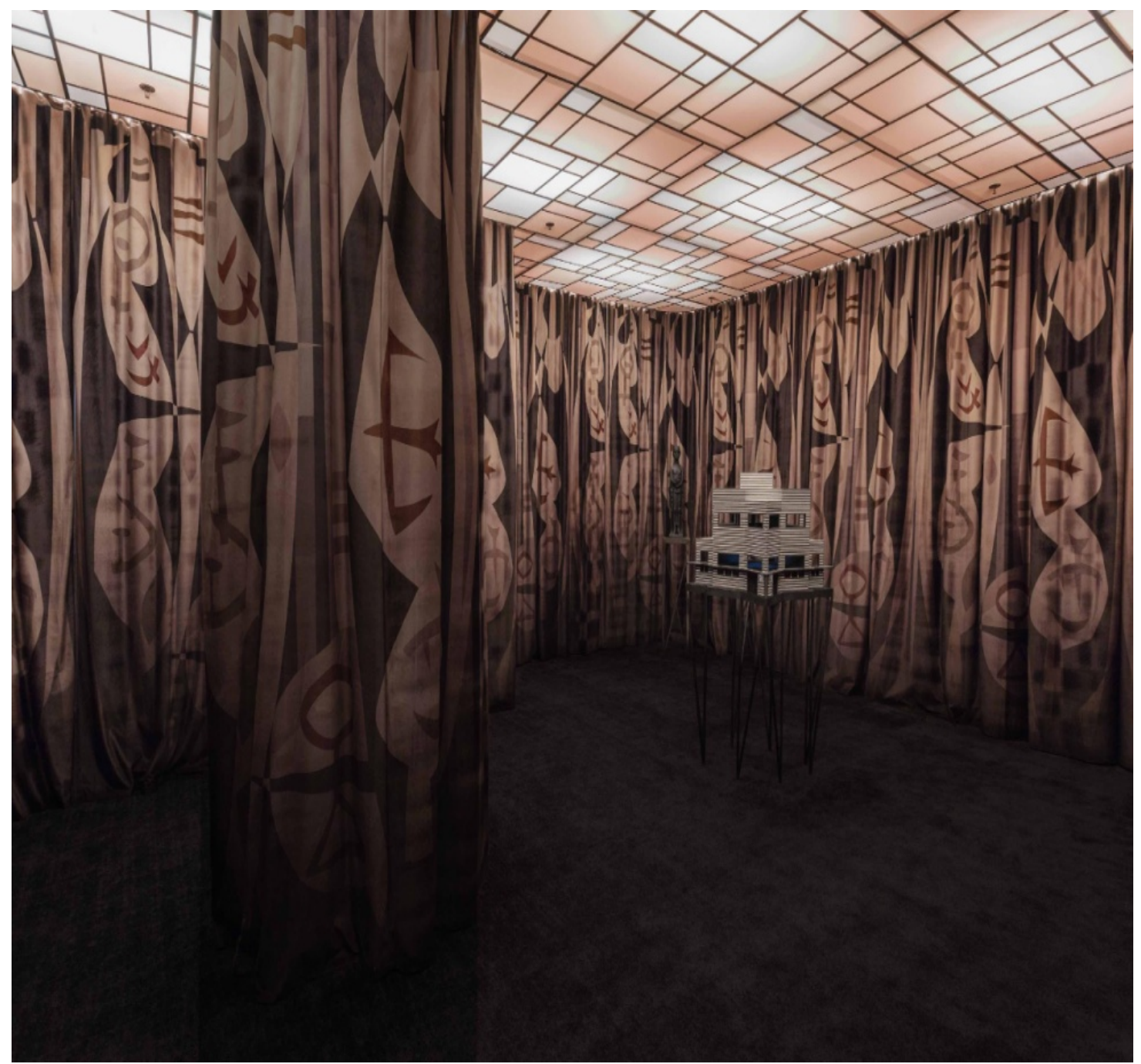

Note. Source : avec la permission de la Fondation PHI pour l'art contemporain Photo : Richard-Max Tremblay 
Figure 2.

The Land of Plenty (2018-2019) de Jasmina Cibic

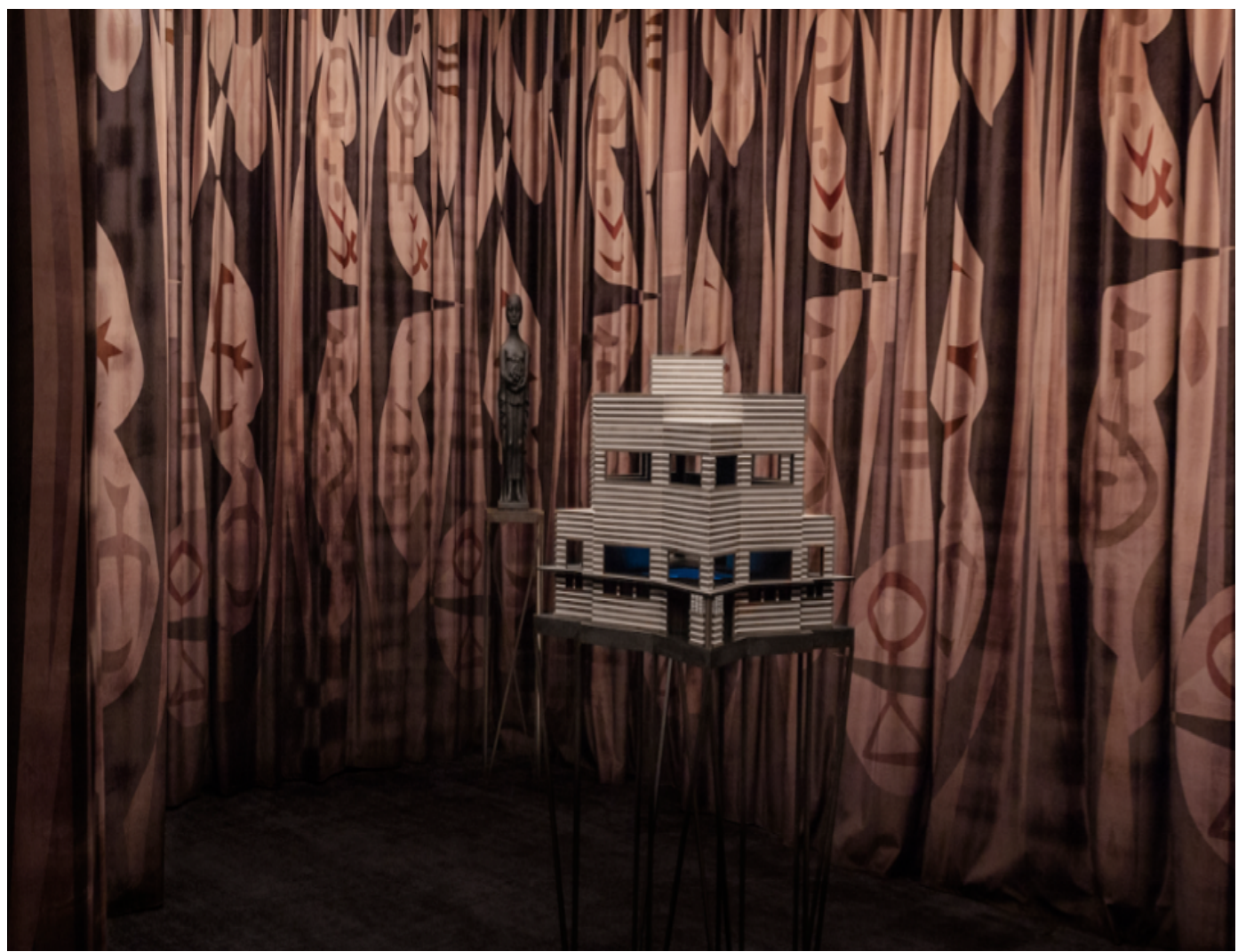

Note. Source : avec la permission de la Fondation PHI pour l'art contemporain Photo : Richard-Max Tremblay

$\mathrm{Au} 4^{\mathrm{e}}$ étage du bâtiment principal, se trouvait l'installation The Epilogue (2019) qui mettait en vedette le pavillon de la Yougoslavie présenté à l'Expo 67 de Montréal. Ce pavillon est le dernier que la Yougoslavie a présenté à une exposition universelle, préfigurant sa dissolution violente dans les années 1990. Cibic a imaginé une installation comportant six photographies de la mère-nation au drapeau, arborant divers slogans politiques, ainsi qu'un film, État d'illusion (2018), qui utilisait la métaphore du spectacle de magie afin de traiter de la disparition d'un État. La mère-nation au drapeau y était ré-imaginée en magicienne-assistante, telle que manipulée par trois figures masculines. Une reproduction petit format du pavillon yougoslave de l'Expo 67 se trouvait sur scène. Ce dispositif était sans cesse démantelé et reconfiguré par les figures masculines, afin d'y donner cours à six tours de magie, toujours de plus en plus violents, où à chaque fois la mère-nation disparaissait, tout comme les six républiques disparues en Yougoslavie. 
Figure 3.

The Epilogue (2019) de Jasmina Cibic

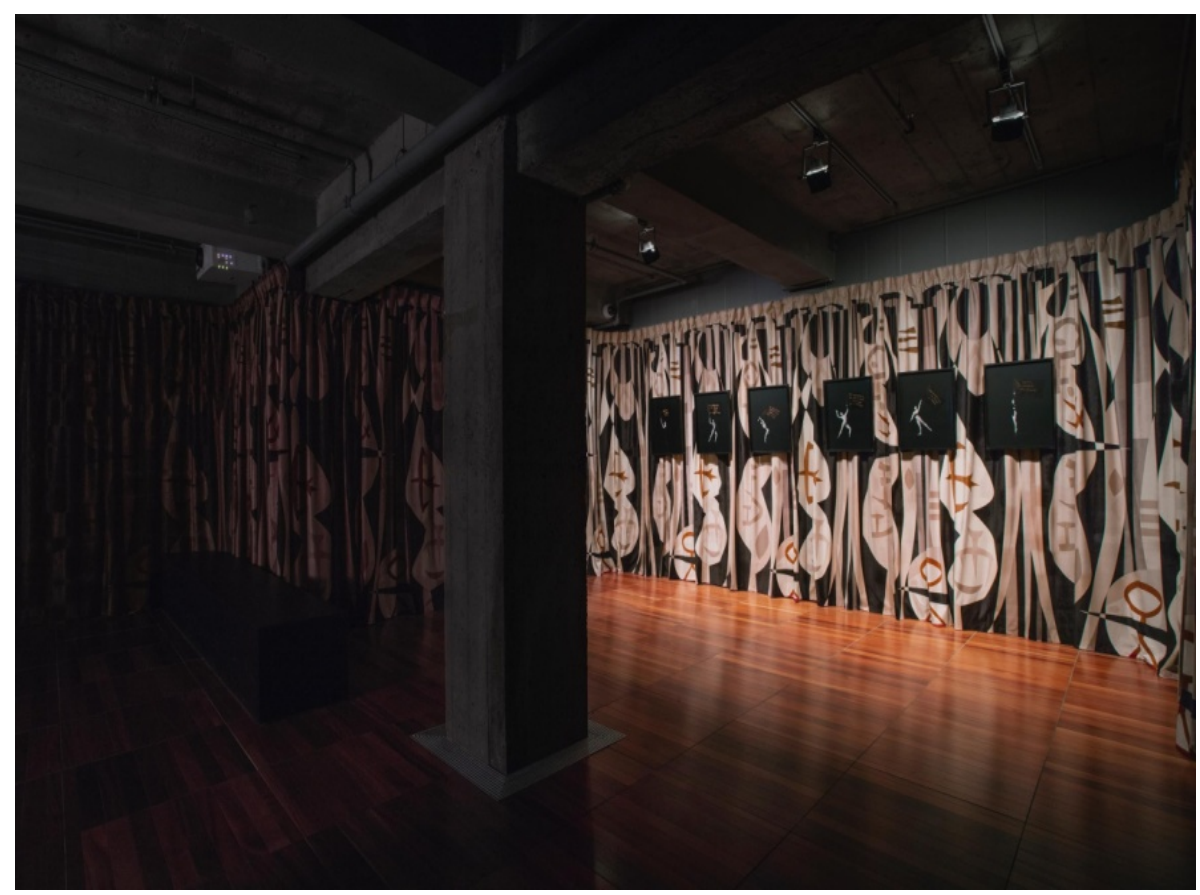

Note. Source : avec la permission de la Fondation PHI pour l'art contemporain Photo : Richard-Max Tremblay

\section{Figure 4.}

The Epilogue (2019) de Jasmina Cibic

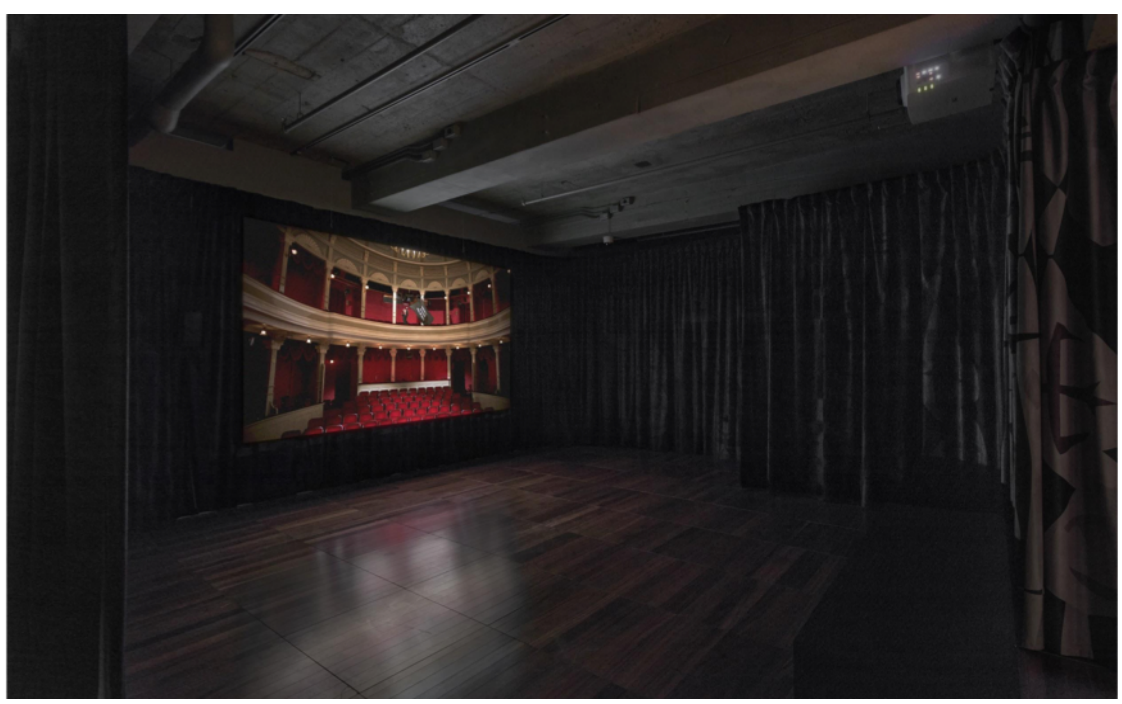


Note. Source : avec la permission de la Fondation PHI pour l'art contemporain Photo : Richard-Max Tremblay

L'installation filmique Nada Act 1 (2016) présentait pour sa part une réflexion au sujet du pavillon de la Yougoslavie présenté à l'Exposition universelle de Bruxelles en 1958. Une des œuvres de l'installation, intitulée Nada (2016), était constituée d'une maquette représentant le pavillon dans sa version originale avec son mât central, qui, selon la vision de son architecte Vjenceslav Richter, devait soutenir une structure suspendue. Ce mât se verra toutefois censuré par le comité de politiciens en charge du projet. Dans l'œuvre Nada Act 1 (2016), le pavillon devient un instrument à cordes joué par la violoniste Dejana Sekulic.

\section{Figure 5.}

Nada : Act 1 (2016) de Jasmina Cibic

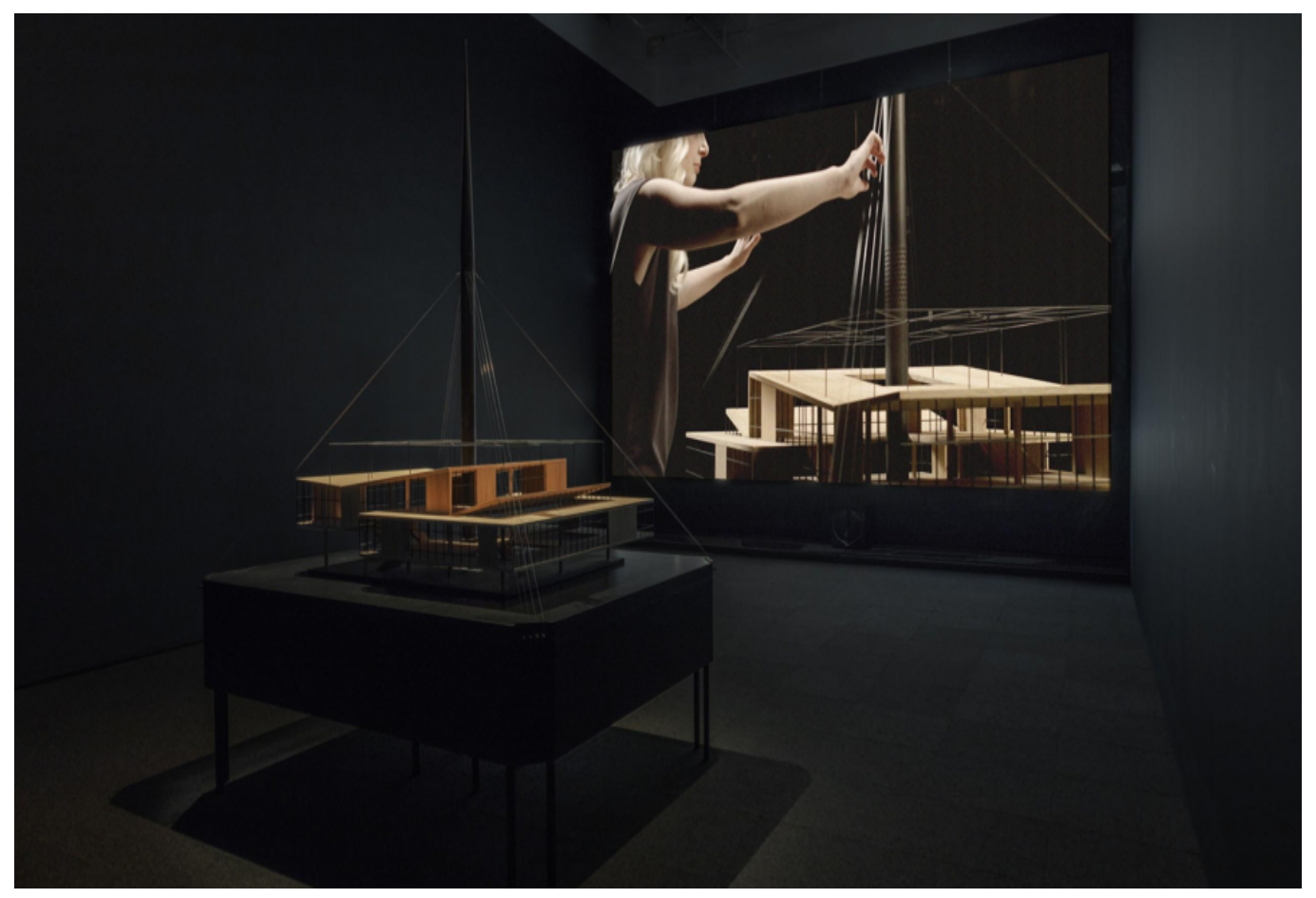

Note. Source : avec la permission de la Fondation PHI pour l'art contemporain Photo : Richard-Max Tremblay

\section{The Land of Plenty : le corps de Joséphine Baker dans l'architecture moderniste}

Revenons maintenant à l'installation The Land of Plenty (2018-2019), qui occupait le premier espace d'exposition du bâtiment au 451 St-Jean. À l'entrée se trouvait, en coin, Land of Plenty (2017), une statuette noire, réplique miniature de la mère-nation au panier de fruits. Celleci était placée, à l'origine en grand format, devant le pavillon moderniste du Royaume des Serbes, des Croates et des Slovènes à l'Exposition universelle de Barcelone en 1929. Cette réplique petit format se fondait dans les rideaux, certain'e·s la remarquaient, d'autres pas tout à fait. 


\section{Figure 6.}

Land of Plenty (2017) de Jasmina Cibic

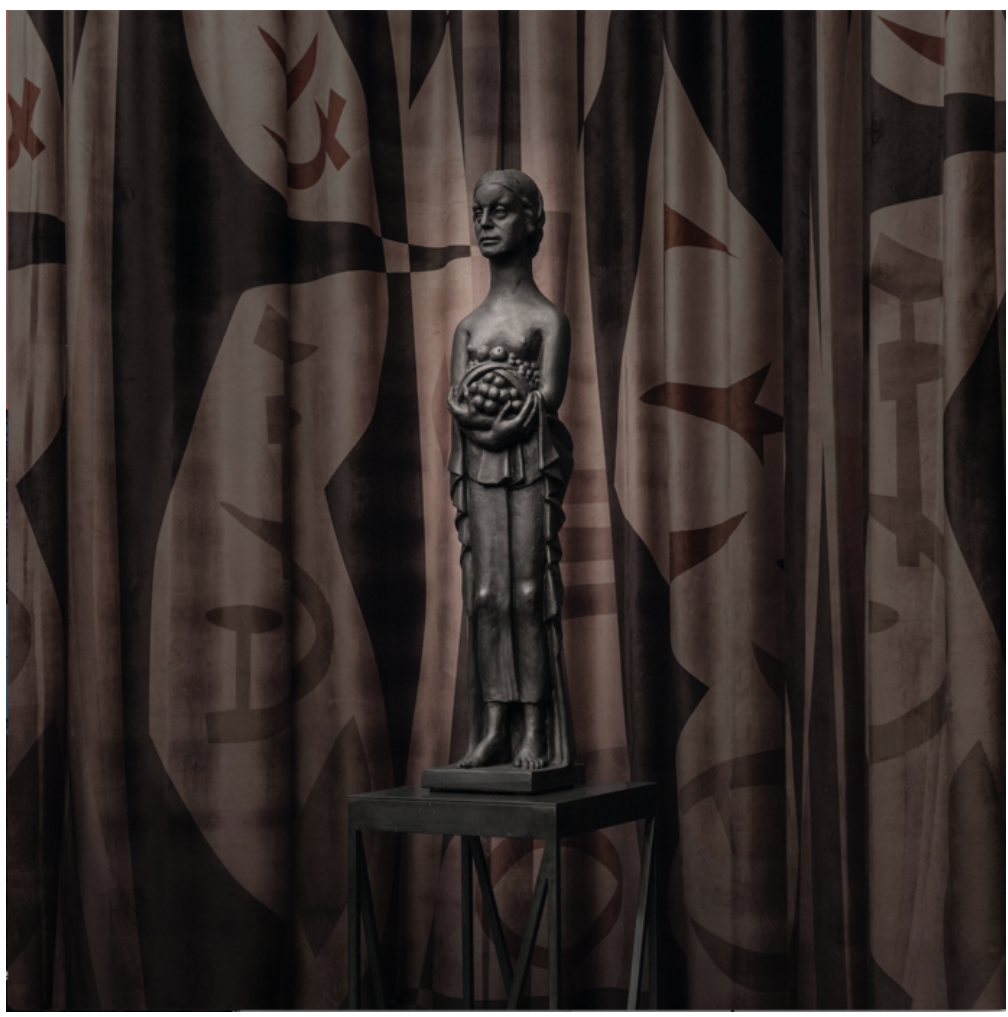

Note. Source : avec la permission de la Fondation PHI pour l'art contemporain Photo : Jasmina Cibic

Ensuite, l'élément central dans l'espace était l'œuvre The Pavillion (2018), une réplique en miniature du Pavillon du Royaume des Serbes, des Croates et des Slovènes. Au centre de la maquette, se trouvait une toute petite piscine bleue illuminée, de la même forme que celle du pavillon. Cachée et secrète, elle existait dans une sorte de marginalité intérieure et souterraine. Cette piscine était une citation architecturale de la villa privée pour la danseuse et performeuse Joséphine Baker, telle que conceptualisée par l'architecte autrichien Adolf Loos en 1928. Cette villa n'a jamais été construite toutefois, elle est restée sous forme de plans. Brašovan s'en est tout de même inspiré pour le design de la façade extérieure à rayures blanches et noires du pavillon national de Barcelone. Cette villa pour Joséphine était conçue de telle sorte qu'elle abritait une piscine intérieure à être utilisée uniquement par Joséphine, accessible par le $2^{\mathrm{e}}$ étage, éclairée par un puits de lumière et entourée de fenêtres derrière lesquelles se trouvaient un boudoir et un café. 


\section{Figure 7.}

The Land of Plenty (2018-2019) de Jasmina Cibic

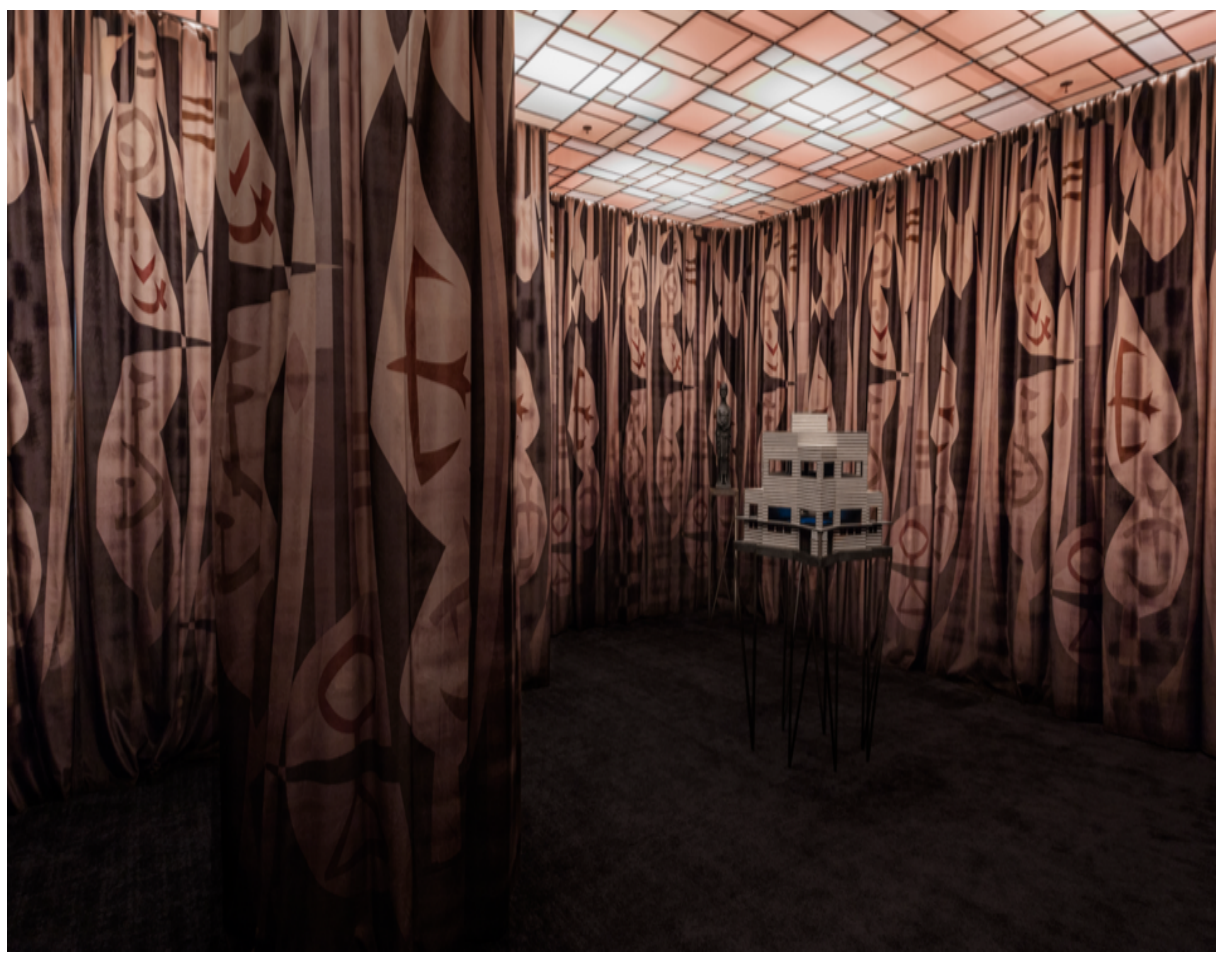

Note. Source : avec la permission de la Fondation PHI pour l'art contemporain Photo : Richard-Max Tremblay

Untitled (2017), le plafond suspendu dans l'installation, était également une citation architecturale, cette fois-ci tirée du plafond moderniste qui se trouvait à l'origine dans l'architecture intérieure de l'ancien palais de la Fédération à Belgrade. De ce plafond émergeait une lumière rose tamisée qui baignait l'espace. Il y avait également au fond ouest de l'espace trois photographies, The Artist (2017), Germania (2017), The Curator (2017), qui représentaient trois allégories féminines manipulant des accessoires abstraits à l'esthétique moderniste. Les rideaux regorgeant d'ornements entouraient toute la pièce, cachant en partie entrée et sortie pour les visiteur·euse·s qui se trouvaient au centre de l'espace. Aussi, tel que déjà mentionné, l'installation était conçue par Cibic comme le boudoir privé d'un collectionneur anonyme. Ce boudoir s'insérait, tel un épiderme, à l'intérieur du Cube blanc, espace public de la Galerie d'art. 


\section{Figure 8.}

Land of Plenty (2018-2019) de Jasmina Cibic

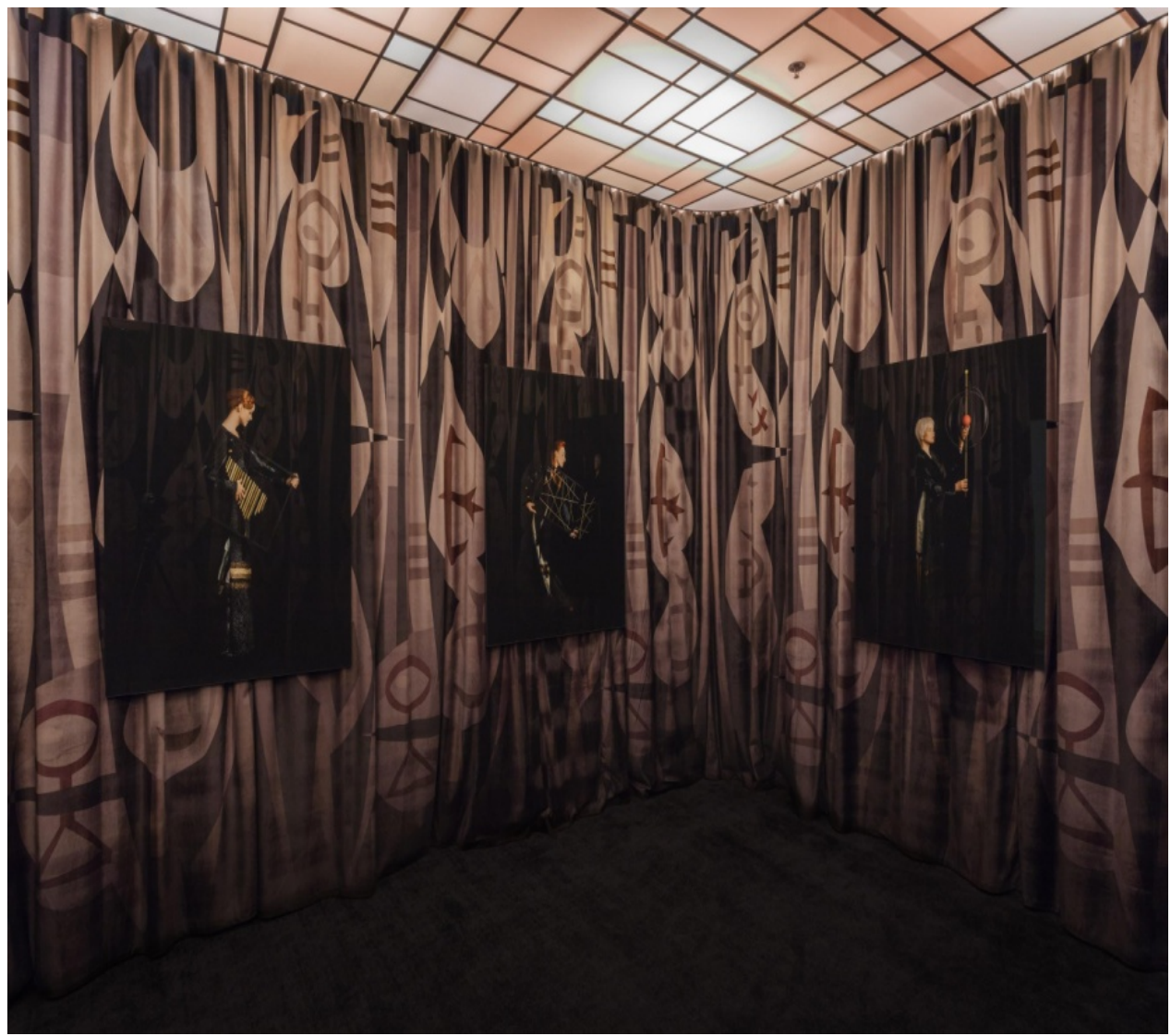

Note. Source : avec la permission de la Fondation PHI pour l'art contemporain Photo : Richard-Max Tremblay

Déambuler dans The Land of Plenty (2018-2019) nous plongeait dans l'exploration d'une installation amalgamant espace public et espace privé, expérience centrale et expérience marginale, forme féminine en tant qu'État nation et forme moderniste en tant qu'État nation, et les enjeux de force de persuasion y étant associés. Ainsi, centrale, se trouvait l'architecture extérieure publique d'un pavillon national issu de l'entreprise patriarcale moderniste. Mais cachée, à l'intérieur, se trouvait cette piscine, apparemment privée, conçue par Adolf Loos pour accueillir le corps nu de Joséphine Baker, nageant dans cet élément liquide et sensuel. La piscine intérieure de Joséphine était éclairée par un puits de lumière et était entourée de vastes fenêtres où, derrière, se trouvaient des espaces de réception, dont un café accueillant des hommes pouvant observer celleci se baigner. Cet espace "privé », la piscine de Joséphine, devient donc public, et un espace public, le pavillon national de Barcelone, devient en partie privé.

\section{Joséphine : écriture poétique, théories féministes de la corporéité et pédagogie}


À première vue, on peut comprendre l'architecture de la piscine intérieure et du café adjacent de Loos comme un dispositif de surveillance, de voyeurisme, d'objectification et d'exotisme pour le corps de Joséphine. Ce qu'il est, en effet. Toutefois, mes recherches explorant les théories féministes de la corporéité - celles de Beatriz Colomina sur les corps féminins dans les intérieurs de Loos (1994), celles de Luce Irigaray sur la « mécanique » des fluides (1977), celles d'Elizabeth Grosz sur les corps volatils (1994) et celles de Vivian Sobchack (2004) sur la pensée charnelle - me permettaient de comprendre que la réalité de l'expérience incorporée de la femme dans l'espace construit par les hommes était plus complexe et ambiguë : une situation limitée, certes, mais aussi une occasion de possibilités. Le corps de Joséphine dans la villa de Loos se nuançait alors. Je me questionnais sans cesse : comment faire pour aborder en visites de groupe le sujet du corps féminin dans les architectures patriarcales de manière à faire honneur à la complexité, aux limites et aux potentialités de la situation ?

Lire les théories féministes de la corporéité enrichissait ma réflexion, mais il y avait pour moi une limite à cet acte de lecture. Bien qu'éclairantes, ces lectures théoriques me plaçaient dans un état de réception, qui n'était pas passif, c'est certain, mais en même temps, pas aussi actif que je l'aurais souhaité. Je me sentais parfois sans ancrage et immergée dans une matière qui était vaste et fouillée. Et puis, bien que j'aie lu les théories, je n'arrivais pas à trouver les outils pédagogiques utiles pour en discuter de manière satisfaisante lors de mes animations de visites de groupe. C'est alors que j'ai ressenti fortement qu'il serait utile pour moi de recommencer à rédiger des écrits poétiques, ici en lien avec les œuvres de l'exposition de Jasmina Cibic, et en particulier pour l'œuvre The Land of Plenty (2018-2019). Lors de mes études doctorales, j'ai incorporé ces écrits dans ma thèse, mais après l'obtention de mon diplôme, je n'ai continué cette pratique que sporadiquement. Dans le flot de mon travail, il est en effet difficile de trouver le temps d'écrire de la poésie. Toutefois, je sentais qu'une clé à mes problèmes pédagogiques autour du sujet de la corporéité féminine dans The Land of Plenty (2018-2019) se situait probablement dans ce processus de rédaction de poèmes. L'un d'eux est devenu Joséphine dans le boudoir de l'architecte. Et mon hypothèse s'est révélée vraie. Cette écriture poétique représente ma façon d'interagir avec les théories féministes de la corporéité, ainsi qu'avec The Land of Plenty (20182019), avec agentivité et créativité. Elle constitue mon ancrage et mes racines par rapport à ces théories et à cette installation. Aussi, elle me permet d'entrevoir une potentielle élaboration fructueuse d'un contenu pédagogique pouvant transmettre la complexité des approches féministes de la corporéité dans le domaine de l'art contemporain aux participant $\cdot e \cdot s$ à mes visites de groupe.

À l'image du processus de recherche de Vivian Sobchack (2009), spécialiste de la phénoménologie du cinéma et de la culture de l'image en mouvement, la cristallisation de mon poème me permet d'interpréter l'installation The Land of Plenty (2018-2019) d'abord par l'entremise de mon expérience vécue. Toutefois, ce n'est pas mon expérience vécue que je théorise. Sobchack affirme que lors de ses interprétations de films, elle commence souvent par sa propre expérience. Ensuite, toutefois, elle généralise celle-ci à des structures d'expériences plus larges que tous et toutes peuvent habiter (p. 2). Mon poème Joséphine dans le boudoir de l'architecte constitue mon geste d'empathie envers l'œuvre The Land of Plenty (2018-2019) en tant que théorisation de ma propre subjectivité au contact de l'œuvre. Aussi, il me permet de m'engager subjectivement face à la théorie féministe de la corporéité, élargissant mon expérience à des structures d'expériences plus générales. Je me pose alors ces questions : comment puis-je développer mon empathie envers la figuration de Joséphine dans la villa de Loos avant d'enseigner mes visites de groupe ? Et comment cette empathie me permet-elle d'enseigner 
autrement lors de mes visites de groupe ? Comment me permet-elle d'utiliser autrement mon langage parlé lors de mon enseignement?

\section{Joséphine dans le boudoir de l'architecte}

Le bassin est petit, elle aimerait l'océan.

Nue, Joséphine nage.

Dans l'illusion, encore

D'avoir troqué

Le strass de la scène

L'éclat du théâtre

Pour l'intime de sa maison.

Le cœur de son bol d'eau.

Nue, Joséphine nage.

Ses épaules, son thorax, ses bras

Au plus loin, du plus loin

S'étirent.

Ludique

Elle culbute.

Fait la planche.

Ses mains, ses jambes, ses pieds

Au plus loin, du plus loin

S'ouvrent.

Elle ferme les yeux.

Immerge doucement sa tête

À l'écoute

De ce silence amniotique

Qu'elle connaît si bien.

Le bassin est petit, elle aimerait l'océan.

Nue, Joséphine nage.

Cernée.

Sa peau, son ventre, ses cuisses, scrutés.

Aveuglée.

Ses seins, son cou, ses cheveux, détaillés.

Tout autour

Une galerie de fenêtres

Derrière

Un boys club

Plusieurs attablés.

Cafés, cognacs, cigares, fumée.

Le bassin est petit, elle aimerait l'océan. 
Nue, Joséphine plonge.

$\mathrm{Au}$ fond de l'eau

Elle cherche une issue.

À tâtons

Une matière

De pierre

Se forme

Se dessine.

Mémoire sous-marine.

Mère-nation,

Statue au panier de fruits.

Icône de tout un pays.

Déesse de l'unification

Arrimée à la proue du navire.

Glorieuse

Elle guide l'équipage

Brandit le drapeau.

Ondulant avec superbe.

Déboulonnée

Délestée

Coulée.

Maintenant

Épave plombée.

Les mains de Joséphine

Caressent la mère au panier de fruits.

Elle ressent, sous ses doigts,

Les aspérités de ses cheveux tressés

Les creux et vallées

De son visage pétrifié

De ses sourcils froncés

De ses yeux durs et blancs.

Lèvres

Bleues.

Étreinte

Gelée.

Le boys club

Est là

Toujours là.

Cafés, cognacs, cigares, fumée. 
Scaphandrière

Elle entre en scène.

À la brasse

À la planche

Elle entre en scène.

La scène est là.

Toujours là.

Dans son salon

Elle entre en scène

Dans sa chambre

Elle entre en scène

Dans son lit

Elle entre en scène.

Dans cette maison

Qui n'a

Jamais été

Sienne.

Elle entre en scène.

Sur les rebords de son bassin.

Gracieuse

Elle s'assied.

Sèche sa peau humide

Allonge

Ses jambes

De marbre.

Le soleil

Plombe

Par le puits de lumière.

Aveuglée

Elle lève

Son bras

D'ébène.

Ombrage

Son regard.

Dans le boudoir feutré

De l'architecte.

Dans le boys club

Des attablés. 
Elle fait son entrée.

La voilà !

Hôtesse splendide.

Uniforme

Seyant.

Fichu

De soie

Au cou

Parfait.

Brillant

À lèvres

Tamisé.

Elle sourit.

À tous ces attablés.

Elle glisse, avec charme et finesse.

De table en table.

Elle se faufile

Attentionnée.

Élégante.

« Du café ?»

«Quelques biscuits?»

«Un trait de brandy?».

«Laissez-moi le soin ... »

«... De nettoyer tout ça. »

«Pardon ...?

«... À quand le prochain numéro ...? »

« Très, très bientôt ».

Mon poème me permet d'explorer plus à fond la subjectivité de Joséphine, alors qu'elle oscille entre possibilités et limites, et qu'elle subit les effets de l'architecture patriarcale sur son corps. Elle se croit d'abord seule et se meut en toute intimité : cette eau dans cette piscine constitue une possibilité de libération privée de son corps, un confort, un plaisir et une détente. Mais bien vite, elle réalise que sa situation est tout autre que ce qu'elle avait pensé au départ : elle ressent et comprend les regards voyeurs des hommes sur elle. Se savoir ainsi observée par des regards cachés la trouble, l'effraie et l'inhibe. Elle est soudain limitée et heurtée par les fenêtres qui l'entourent et par tous ces hommes fixés sur son corps. C'est alors qu'elle plonge dans l'eau pour échapper à cette situation. Elle parvient à repousser les limites que lui impose le bassin. Par les mouvements de son corps et de ses bras elle réussit l'impossible : ouvrir, sous la surface, une profondeur océanique. Elle va jusqu'à atteindre les fonds marins. C'est alors qu'elle découvre la mère de pierre, épave gelée. Un écho de la mère-nation au panier de fruits du premier pavillon de la Yougoslavie à l'Exposition universelle de Barcelone. Ensuite, les images et le langage du poème nous mènent à la transformation de cette mère-nation en une mère plus intime et possiblement très proche de Joséphine : celle qui a enfanté, qui a produit des fruits, et qui est maintenant abandonnée, 
pétrifiée, coulée. C'est alors que Joséphine fait la paix, par la caresse et par l'étreinte, avec cette mère : un rituel de tendresse, de deuil et d'acceptation.

À ce même moment, Joséphine réalise qu'elle est encore observée, que même dans ces profondeurs, elle est encore sur scène, sans cesse. À cet effet, l'historienne et théoricienne féministe de l'architecture Beatriz Colomina (1994) nous indique que les intérieurs d'Adolf Loos prennent la forme à la fois d'une architecture de la matrice et d'une architecture théâtrale. La piscine intérieure de Baker en est un exemple probant : ce bol d'eau transparent où le corps de celle-ci est immergé, qui rappelle le liquide amniotique et l'utérus de la mère, mais aussi ces fenêtres, qui entourent l'espace aqueux et sensuel, qui représentent la dimension théâtrale de cette architecture. Joséphine va graduellement habiter et prendre le contrôle de ces deux composantes architecturales de la villa, ce qui lui conférera force et pouvoir d'agir. Maintenant qu'elle a apaisé son rapport à la mère, Joséphine comprend quelque chose de manière plus consciente au sujet de l'abandon et du rejet du corps maternel dans nos sociétés et cultures. C'est donc riche d'une lucidité et d'une agentivité nouvelles qu'elle remonte à la surface et, par le mouvement de son corps, elle a maintenant une compréhension plus profonde et renouvelée du dispositif architectural phallocentrique. Car c'est par son corps d'abord que sa conscience s'éveille : elle remonte à la surface, elle sort de l'eau, elle traverse la paroi qui la sépare des hommes, elle entre dans le boudoir des attablés et ce, maintenant, en toute connaissance de cause. Il y a alors une ambiguïté et une possibilité qui se dessinent: de nageuse, Joséphine devient l'hôtesse, elle est au service des attablés, mais aussi elle promet à ceux-ci un prochain spectacle. La fin est ouverte : on peut imaginer qu'elle proposera, dans le futur, un spectacle selon ses propres paramètres. Elle sera, possiblement et dorénavant, la chorégraphe, la metteure en scène et la performeuse du spectacle.

La philosophe et théoricienne féministe Elizabeth Grosz nous rappelle que dans les sociétés patriarcales le corps de la femme a été construit, non seulement comme une absence ou un manque, mais de manière plus complexe comme un liquide incontrôlable qui fuit et qui s'infiltre, un flux sans forme (1994, p. 203). Cette conception patriarcale « du corps féminin qui fuit » est considérée comme abjecte et monstrueuse. Grosz propose alors comme projet féministe de se réapproprier cette conception dévaluée du corps de la femme pour en faire, de manière subversive, une force : un flux créatif, désirant, fructueux et constructif. Dans ce même esprit, la philosophe et psychanalyste féministe Luce Irigaray propose, dans la « mécanique » des fluides, de mettre de l'avant le fluide afin de contester l'hégémonie du scopique. Selon elle, les fluides sont la trace résiduelle de la relation primaire au maternel. Elle théorise les fluides pour contrer l'oubli des fondations maternelles dans les discours philosophiques et psychanalytiques phallocentriques. Ainsi, Irigaray ouvre un espace pour contribuer au projet féministe de ré-imaginer la subjectivité féminine (1977). Selon elle, il est impossible pour les femmes de revenir à un corps hors de sa représentation dans la culture patriarcale. Ainsi, le corps de la femme est soumis aux forces d'oppression jusque dans son existence dans l'espace pré-linguistique de l'imaginaire. Toutefois, Irigaray met au défi l'inévitabilité de ces formes conventionnelles dans l'espace de l'imaginaire et du symbolique. Elle argumente que nous devons et pouvons reconstruire un espace imaginaire et un espace symbolique du corps de la femme qui soient positifs pour celle-ci. Le travail de la théoricienne est un travail créatif au cœur duquel le corps de la femme est ré-imaginé, avec amour et soin, et réarticulé pour permettre aux femmes de ressentir et de penser différemment leur forme incorporée. La « mécanique » des fluides suggère une ambiguïté du corps féminin, une fluidité, une mobilité, un rejet des formes stables.

Dans le poème, le monde de Joséphine est empli de manifestations liquides : eau du bassin, liquide amniotique, océan. De plus, tous ses gestes et mouvements se succèdent, telle une 
chorégraphie fluide, elle habite le monde sur un mode tactile et mobile : elle nage, s'étire, elle plonge, à tâtons au fond de l'eau elle découvre la mère, l'étreint, elle remonte à la surface, elle sort du bassin, se faufile dans le boudoir, glisse de table en table. Dans le poème, l'épave de pierre morte et gelée au fond des eaux évoque la solidification du corps de la mère, la répression et le déni des fluides comme base pré-linguistique de la philosophie phallocentrique. L'oscillation du corps de Joséphine, entre statue de marbre et d'ébène et corps réel vibrant, fluide et mobile, évoque aussi cette lutte contre la congélation phallocentrique des fluides (Irigaray, 1974). Malgré les obstacles solides qu'elle rencontre, Joséphine les surpasse et est constamment en mouvement. Et quand elle risque la pétrification, par le contact avec la mère, et ensuite par la sortie du bassin et le risque de devenir statue, elle y échappe : d'abord par sa caresse et son étreinte du corps de la mère, ensuite par sa volonté de sortir de l'eau, de se lever et d'entrer dans le boudoir des hommes pour jouer le jeu selon ses propres règles. Elle déleste son corps du poids de la statue de marbre et d'ébène pour devenir-autre en se mettant en marche pour entrer dans le boudoir de l'architecte. Elle y glisse et s'y déplace en proximité avec les attablés. Elle est maintenant leur hôtesse, mais. comme mentionné plus haut, on sent qu'en elle naît une défiance : elle prépare une autre sorte de spectacle, cette fois-ci, en pleine connaissance de son pouvoir de subversion.

\section{Un tournant vers les visites poétiques performatives}

À cette étape de ma réflexion, sur la manière dont j'aurais pu utiliser le poème Joséphine dans le boudoir de l'architecte dans le cadre de mes animations de visites dans The Land of Plenty (2018-2019), j'imagine quelques visites de groupes performatives axées sur la poésie. J'ai déjà écrit deux autres poèmes en lien avec deux autres installations de l'artiste : La déesse pantomime, mon interprétation de l'installation The Epilogue (2019), ainsi que His Pavilion with Strings: Her

Seismic Violin, mon interprétation de l'œuvre Nada : Act 1 (2016). J'imagine donc un parcours où je guide un groupe à travers les trois installations ci-haut en lisant mes poèmes pour ces trois œuvres à chaque étape du trajet. En tant que poète pédagogue, mon désir serait alors, après la lecture du poème, d'animer une conversation avec les visiteur'euse's au sujet à la fois des œuvres et des poèmes. Je n'analyserais pas systématiquement mon poème avec le groupe, comme je viens de le faire ci-haut, à l'aide des théories de Colomina, Grosz, Irigaray et Sobchack. Toutefois, j'aurais au préalable effectué cette analyse de mes poèmes. Et puis, étant donné que la théorie féministe de la corporéité se concentre cette fois-ci sur mes écrits poétiques, celle-ci devient fructueusement ancrée et enracinée en moi. C'est donc forte de cet ancrage que j'animerais la conversation qui suivra l'expérience de l'œuvre et l'expérience de mon poème. J'imagine pour ces visites poétiques performatives tout type de groupes comme public.

J'imagine également un autre type de visite de groupe poétique que j'aurais pu potentiellement animer dans l'exposition de Jasmina Cibic, cette fois-ci une visite-atelier d'écriture poétique pour des publics ciblés d'élèves ou d'étudiant·e·s intéressé·e·s par cette pratique. J'écrirais toujours moi-même des poèmes afin de me préparer à l'animation de ces visites-ateliers, toutefois le cœur de l'activité en déambulation serait la rédaction collaborative de poèmes spontanés par les participant·e·s. Ici, Olga M. Hubard (2007), pédagogue féministe de la corporéité en éducation à l'art muséale, est une inspiration. Celle-ci développe diverses activités tactiles et somatiques lors de la visite de groupe dans l'exposition d'art contemporain, dont des activités de composition collective de poèmes en réponse à l'art exposé. 


\section{Conclusion}

Cette recherche sur ma méthode des concepts en mouvement pour une pédagogie poétique pour la visite de groupe m'a permis de réfléchir à cette idée du devenir-autre pour l'éducatrice muséale à l'art contemporain. Mon poème Joséphine dans le boudoir de l'architecte a été l'occasion de plonger profondément et intimement au cœur de l'œuvre The Land of Plenty (20182019), et de me servir des mots comme matières résonantes pour exprimer cette exploration. Également, en dialogue avec les théories féministes de la corporéité, ce poème m'a permis d'élargir mon expérience pour généraliser celle-ci à des structures d'expériences plus larges, que tous et toutes peuvent habiter, au sujet du corps féminin dans les architectures patriarcales (Colomina, 1994 ; Grosz, 1994 ; Irigaray, 1977 ; Sobchack, 2009).

Au sujet de l'éthique de la potentialité, Giorgio Agamben affirme : « (...) this is why the only ethical experience (which, as such, cannot be a task or a subjective decision) is the experience of being (one's own) potentiality, of being (one's own) possibility - exposing, that is, in every form one's own amorphousness and in every act one's own inactuality » (2013 [1993], p. 43). Toutefois, à mon avis, cette éthique de l'amorphe et de l'informe, qui permet de dé-solidifier corps, choses et environnement pour tendre vers un devenir-autre, crée possiblement une expérience incorporée déracinée, à la dérive et éthérée. Ce que je propose plutôt, c'est le geste éthique féministe de l'écriture poétique pour créer une forme cristallisée. Ce cristal porte en lui, tout à la fois, la fluidité, la mobilité, les forces et les intensités rhizomatiques du corps-sujet, de l'espace environnant, et des choses, et il offre également une complexe géométrie kaléidoscopique organisée, structurée et cernée par la surface irrégulière du cristal. Par celui-ci, il est possible de dépasser l'état d'entre-deux du devenir-autre pour accéder au corps-sujet. Et voilà que ma recherche dans l'exposition de Jasmina Cibic renforce cette conviction : Joséphine dans le boudoir de l'architecte, La déesse pantomime et His Pavilion with Strings: Her Seismic Violin correspondent pour moi à cette cristallisation qui façonne mon corps-sujet. D'où l'importance vitale de poursuivre cette pratique d'écriture poétique pour ma pratique pédagogique (Prendergast et al., 2009). Car celle-ci constitue le cœur, les racines, l'ancrage et l'élan de revitalisation de mon programme pédagogique pour la visite de groupe dans l'exposition d'art contemporain.

\section{Références}

Agamben, G. (2013, [1993]). The coming community. University of Minnesota Press.

Agamben, G. (1999). Potentialities: Collected essays. Stanford University Press.

Bal. M. (2002). Travelling concepts in the humanities: A rough guide. University of Toronto Press.

Bal, M. (2001). Louise Bourgeois' spider. The architecture of art-writing. The University of Chicago Press.

Beattie, A., Fiset, D., Keenlyside, E., Lemaire, M-H., \& Pyne Feinberg, P. (2018). Embodied dialogue: Group learning and collaboration at DHC/ART Foundation for Contemporary Art. Muséologies, 9(1), 135-160. https://doi.org/10.7202/1052632ar 
Colomina, B. (1994). Privacy and publicity: Modern architecture as mass media. MIT Press.

Deleuze, G. \& Guattari, F. (2005 [1991]). Qu'est-ce que la philosophie? Les Éditions de Minuit. Flusser, V. (2014). Gestures. University of Minnesota Press.

Grosz, E. (1994). Volatile bodies. Towards a corporeal feminism. Indiana University Press.

Grosz, E. (1995). Space, time and perversion. Routledge.

Hubard, O. M. (2007). Complete engagement: Embodied responses in art museum education. Art Museum Education, 60(6), 46-53. https://doi.org/10.1080/00043125.2007.11651133

Irigaray, L. (1974). Speculum de l'autre femme. Les Éditions de Minuit.

Irigaray, L. (1977). Ce sexe qui n'en est pas un. Les Éditions de Minuit.

Prendergast, M., Leggo, C., \& Sameshima, P. (2009). Poetic inquiry: Vibrant voices in the social sciences. Sense Publishers.

Sobchack, V. (2009). Vivian Sobchack in conversation with Scott Bukatman. E-Media Studies, 2(1), 1-11. https://dx.doi.org/10.1349/PS1.1938-6060.A.338 